\title{
Experimental studies to improve specific actinide decay data
}

\author{
F.G. Kondev ${ }^{1, \mathrm{a}}$, M.A. Kellett ${ }^{2}$, I. Ahmad $^{3}$, J.P. Greene ${ }^{3}$, and A.L. Nichols ${ }^{2}$ \\ 1 Nuclear Engineering Division, Argonne National Laboratory, Argonne, IL 60439, USA \\ 2 Nuclear Data Section, Department of Nuclear Sciences and Applications, International Atomic Energy Agency, 1400 Vienna, Austria \\ ${ }^{3}$ Physics Division, Argonne National Laboratory, Argonne, IL 60439, USA
}

\begin{abstract}
A Co-ordinated Research Project (CRP) entitled "Updated Decay Data Library for Actinides" was initiated in 2005 by the International Atomic Energy Agency (IAEA), with the primary aim of improving the quality of recommended decay data for actinides and their decay chains. Participants have attended two Research Coordination Meetings, and agreed on a list of important nuclides and evaluation methodologies to be adopted in work programmes dedicated to the preparation of a new database. Specific measurements were also proposed to address discrepancies between existing data, and some of these experimental studies are described in detail within this paper.
\end{abstract}

\section{Introduction}

Accurate decay data for actinide nuclides and their decay chains are important in the nuclear fuel cycles of both thermal and fast reactors, including nuclear facility design, fuel manufacture and reprocessing, waste storage and management, safety assessments, provision of safeguards, and quantifying the conditions and monitoring needs for nonproliferation. Following recommendations of an Advisory Group Meeting on Transactinium Isotope Nuclear Data [1], the Nuclear Data Section of the International Atomic Energy Agency organized a Coordinated Research Project (IAEACRP) in 1977 to measure and evaluate the decay data for a number of actinide nuclides and their decay chains. This effort resulted in the assembly of a dedicated library that included detailed evaluated decay data for 23 nuclides and tables of recommended half-lives, branching fractions, and $\gamma$-ray and $\alpha$-particle energies and emission probabilities for a larger number of transactinium nuclides and their radioactive daughters [2].

Further extensive experimental studies have been carried out over the past thirty years in the region of the heavy and super-heavy nuclei (see ref. [3] and references therein), driven mainly by the needs of the nuclear structure community. These data need to be assessed and evaluated in a consistent manner prior to their inclusion in the various nuclear applications libraries. Furthermore, well-defined requirements for improved actinide decay data have been specified in a recent review by Nichols [4]. As a consequence of this review and subsequent debate within the nuclear data community, an IAEA-CRP on "Updated Decay Data Library for Actinides" was begun in October 2005. The selected radionuclides are noted in this paper, along with the results from specific measurement activities. A detailed overview of the IAEACRP and specific data evaluation activities are presented elsewhere [5].

\footnotetext{
${ }^{a}$ Presenting author. Tel.: +1 (630) 252-4484; Fax: +1 (630) 252-
} 4978; e-mail: kondev@anl.gov
Table 1. Radionuclides selected for re-evaluation.

\begin{tabular}{ll}
\hline Actinides & Natural decay products \\
\hline${ }^{228,229,231,232,233,234} \mathrm{Th}$ & ${ }^{206} \mathrm{Hg}$ \\
${ }^{231,233} \mathrm{~Pa}$ & ${ }^{206,207,208,209,210} \mathrm{Tl}$ \\
${ }^{232,233,234,235,236,237,238,239} \mathrm{U}$ & ${ }^{209,210,211,212,214} \mathrm{~Pb}$ \\
${ }^{236,236 m, 237,238,239} \mathrm{~Np}$ & ${ }^{210,211,212,213,214,215} \mathrm{Bi}$ \\
${ }^{238,239,240,241,242} \mathrm{Pu}$ & ${ }^{210,211,212,213,214,215,216,218} \mathrm{Po}$ \\
${ }^{241,242,242 m, 243,244,244 m} \mathrm{Am}$ & ${ }^{211,215,217,218,219} \mathrm{At}$ \\
${ }^{242,243,244,245,246} \mathrm{Cm}$ & ${ }^{217,218,219,220,222} \mathrm{Rn}$ \\
& ${ }^{221,223} \mathrm{Fr}$ \\
& ${ }^{223,224,225,226,228} \mathrm{Ra}$ \\
\hline
\end{tabular}

\section{Coordinated Research Project}

Scientists from several research laboratories in eight countries are involved in organized efforts to quantify with greater accuracy the complete decay data for approximately 40 actinides and 45 of their daughter decay products under the auspices of the IAEA-CRP "Updated Decay Data Library for Actinides". A more extensive set of recommended decay data will also be produced to include additional actinides and their decay products from other reputable sources. This project is coordinated by M.A. Kellett of the IAEA Nuclear Data Section, and two Research Coordination Meetings have already taken place at IAEA Headquarters in Vienna [6,7].

After the completion of the project, the resulting evaluated data files will be made available in both ENSDF and ENDF-6 formats so that they can be promptly incorporated into the specialized data libraries used by the applied nuclear community. The list of nuclides selected for detailed study by IAEA-CRP participants is presented in table 1 .

\section{Recent measurements}

Following recommendations made at the first IAEA-CRP meeting [6], measurements on half-lives, and $\gamma$-ray and 


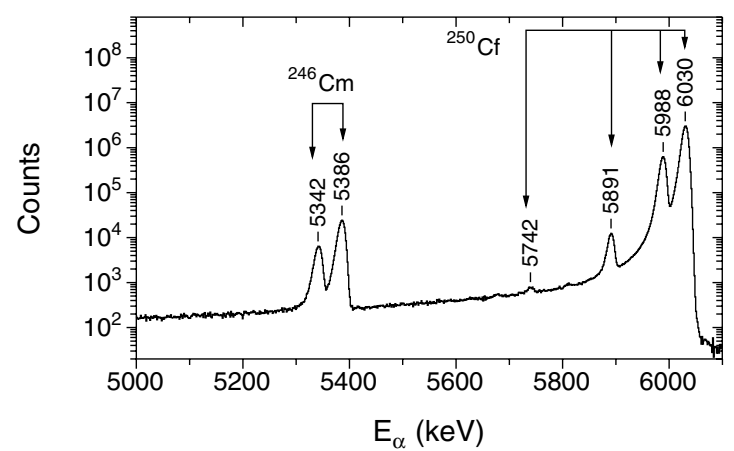

Fig. 1. Alpha-particle spectrum of a mass separated ${ }^{250} \mathrm{Cf}$ source measured with a $25 \mathrm{~mm}^{2}$ PIPS detector at a geometry of $\Omega=0.23 \%$ (numbers indicate the $\alpha$-peak energies in $\mathrm{keV}$ ) - accumulation time was $88.9 \mathrm{~h}$.

$\alpha$-particle emission probabilities for selected nuclides of interest to the IAEA-CRP were carried out at Argonne National Laboratory. Some of the results have already been published [8,9]. Thin and extremely high isotopic purity sources were used that had been produced in the early 1970 s by deposition of $\sim 200 \mathrm{eV}$ ions from a decelerated beam on to thin Al foils using an electromagnetic isotope separator [10]. These sources were stored in Petri dishes and kept at room temperature for more than 20 years. Recently, $\alpha$-particle spectra from the ${ }^{249,250} \mathrm{Cf}$ and ${ }^{244,245,246} \mathrm{Cm}$ samples were measured under a vacuum of $\sim 2 \times 10^{-3}$ torr with Passivated Implanted Planar Silicon (PIPS) detectors of various sizes. Typical leakage currents were $1 \mathrm{nA}$ and the energy resolution of the spectrometers was $10 \mathrm{keV}$ [full width at half maximum (FWHM)]. A measured spectrum of the ${ }^{250} \mathrm{Cf}$ source is shown in figure 1 . Gamma-ray spectra produced from a mass-separated ${ }^{237} \mathrm{~Np}$ source were also measured at various geometries using $2 \mathrm{~cm}^{2} \times 1 \mathrm{~cm}$ LEPS $(\mathrm{FWHM}=0.5 \mathrm{keV}$ at $122.06 \mathrm{keV})$ and $25 \% \mathrm{Ge}(\mathrm{FWHM}=1.8 \mathrm{keV}$ at $1132.51 \mathrm{keV})$ detectors. The energy and efficiency calibrations of these spectrometers were determined with a calibrated mixed-source containing ${ }^{57,60} \mathrm{Co}$, ${ }^{85} \mathrm{Sr},{ }^{88} \mathrm{Y},{ }^{109} \mathrm{Cd},{ }^{113} \mathrm{Sn},{ }^{137} \mathrm{Cs},{ }^{139} \mathrm{Ce}$ and ${ }^{203} \mathrm{Hg}$, as well as monoisotopic sources of ${ }^{152} \mathrm{Eu},{ }^{182} \mathrm{Ta}$ and ${ }^{241,243} \mathrm{Am}$. Special attention was directed towards the energy region below $\sim 100 \mathrm{keV}$, where typical uncertainties for the efficiency of the LEPS spectrometer were determined to be below $2 \%$.

\subsection{Half-lives of long-lived ${ }^{240} \mathrm{Pu},{ }^{245} \mathrm{Cm}$ and ${ }^{246} \mathrm{Cm}$ nuclides}

The accurate determination of the half-life of a very longlived radionuclide is a significant challenge, since direct measurements of the time-dependence of any $\alpha$-, $\beta$-particle and $\gamma$-ray decay signatures are impractical. Experiments are usually aimed at measuring the specific activity of a sample by utilizing either absolute or relative methods. This approach requires the determination of the mass and disintegration rate of the radionuclide with good accuracy. Although mass measurement techniques have been developed to determine isotopic ratios very accurately, there are inherent sources of uncertainty in these methods. For example, absolute efficiencies are needed when determining the mass and activity of a sample, and in the case of $\alpha$-decaying nuclide this type of measurement requires the use of thin and isotopically pure alpha sources, accurate measurement of the solid angle and quantification of the effects of alpha-electron summing, alpha scattering and self-absorption. Furthermore, when a relative specific activity method is used, the half-life of the reference nuclide must be known with good accuracy, and additional uncertainty is introduced.

Another method that can be used to determine the half-life of a very long-lived nuclide is to measure daughter growth in a sample where the shorter-lived parent is present, and therefore the absolute efficiencies are not needed. However, since the technique is relative, the half-life of the parent nuclide must be known with good accuracy. As a test of the in-growth technique, we have determined the half-life of ${ }^{240} \mathrm{Pu}$, which is well defined $[11,12]$. Using the measured ratio $(\mathrm{R})$ of the in-growth ${ }^{240} \mathrm{Pu}$ activity $\left(A\left({ }^{240} \mathrm{Pu}\right)\right)$ to that of the parent ${ }^{244} \mathrm{Cm}$ nuclide $\left(A\left({ }^{244} \mathrm{Cm}\right)\right)$ from the spectrum shown in figure 2 and the known and much shorter half-life of ${ }^{244} \mathrm{Cm}\left(T_{1 / 2}=18.11\right.$ (3) $\mathrm{yr}[13])$, the ${ }^{240} \mathrm{Pu}$ half-life can be determined by means of the equation:

$$
R=\frac{A\left({ }^{240} P u\right)}{A\left({ }^{244} C m\right)}=\frac{\lambda_{d}}{\left(\lambda_{d}-\lambda_{p}\right)} \times\left(1-\exp ^{-\left(\lambda_{d}-\lambda_{p}\right) \times t_{c}}\right)
$$

where $\lambda=\ln 2 / T_{1 / 2}$ is the decay constant, symbols $d(p)$ represent daughter (parent), and $t_{c}$ is the time interval after mass separation when the daughter activity grows into the sample. We have obtained a value of $T_{1 / 2}\left({ }^{240} \mathrm{Pu}\right)=6545$ (19) $\mathrm{yr}$ (see table 2 for details), in excellent agreement with that of 6564 (11) yr, recommended by the US Half-Life Evaluation Committee [11] and the most recently evaluated value of 6561 (7) yr [12]. Using $\alpha$ spectra from the ${ }^{249} \mathrm{Cf}$ and ${ }^{250} \mathrm{Cf}$ (see fig. 1), we have obtained $\alpha$-decay half-lives of 8245 (70) yr and 4706 (40) yr for ${ }^{245} \mathrm{Cm}$ and ${ }^{246} \mathrm{Cm}$, respectively (see table 2). The ${ }^{245} \mathrm{Cm}$ half-life is in a good agreement with a value of 8265 (180) yr reported by Metta et al. [15], but is somewhat lower than the values of 8532 (53) yr and 8445 (200) yr reported by MacMurdo et al. [16] and Polyukhov et al. [17], respectively. Similarly, the deduced half-life of ${ }^{246} \mathrm{Cm}$ is shorter than the results of MacMurdo et al. (4820 (20) yr [16]) and Polyukhov et al. (4852 (76) yr [18]), but in very good agreement with the half-life measurements of Metta et al. (4711 (22) yr [15]) and McCracken et al. (4654 (40) yr [19]).

We have carefully examined potential sources of systematic uncertainties that can be attributed to the in-growth activity technique. The presence of any ${ }^{240} \mathrm{Pu}$ and ${ }^{245,246} \mathrm{Cm}$ activities in the ${ }^{244} \mathrm{Cm}$ and ${ }^{249,250} \mathrm{Cf}$ samples at $t_{c}=0$ is highly unlikely, given the superiority of the mass separation procedure. Loss of ${ }^{240} \mathrm{Pu}$ and ${ }^{245,246} \mathrm{Cm}$ due to the small recoil energy (less than $100 \mathrm{keV}$ ) following $\alpha$-decay of the ${ }^{244} \mathrm{Cm}$ and ${ }^{249,250} \mathrm{Cf}$ parent nuclides is also negligible. One additional point of note is that the sources were not kept under vacuum for long periods of time, but were only used occasionally for short alpha counting measurements. The possibility that the daughter nuclide could escape was checked indirectly using $\gamma$-ray counting measurements with a high-purity germanium detector and a thin, mass separated ${ }^{243} \mathrm{Am}$ source, where the ratio of the $74.66-\mathrm{keV} \gamma$-ray $\left({ }^{239} \mathrm{~Np}\right)$ intensity to that of 
Table 2. Growth time $\left(t_{c}\right)$, daughter $(d) /$ parent $(p) \alpha$-decay activity ratios $(R)$, and the deduced half-lives of ${ }^{240} \mathrm{Pu}$ and ${ }^{245,246} \mathrm{Cm}$; uncertainties are given in parantheses, and are expressed in terms of the last significant digits.

\begin{tabular}{cccrccc}
\hline$t_{c}, \mathrm{yr}$ & \multicolumn{1}{c}{$R, \%$} & parent & $T_{1 / 2}(p), \mathrm{yr}$ & reference & daughter & $T_{1 / 2}(d), \mathrm{yr}$ \\
\hline 32.6137 & $0.6860(11)$ & ${ }^{244} \mathrm{Cm}$ & $18.11(3)$ & {$[13]$} & ${ }^{240} \mathrm{Pu}$ & $6545(19)$ \\
28.6445 & $0.2474(20)$ & ${ }^{249} \mathrm{Cf}$ & $350.6(21)$ & {$[14]$} & ${ }^{245} \mathrm{Cm}$ & $8245(70)$ \\
27.8532 & $0.9359(17)$ & ${ }^{250} \mathrm{Cf}$ & $13.08(9)$ & {$[15]$} & ${ }^{246} \mathrm{Cm}$ & $4706(40)$ \\
\hline
\end{tabular}

Table 3. Alpha-decay energies $\left(\mathrm{E}_{\alpha}\right)$ and emission probabilities $\left(\mathrm{P}_{\alpha}\right)$ of ${ }^{246} \mathrm{Cm}$ and ${ }^{250} \mathrm{Cf}-$ results from the present work and previous measurements; uncertainties are given in parantheses, and are expressed in terms of the last significant digits.

\begin{tabular}{|c|c|c|c|c|c|c|c|c|}
\hline \multirow[b]{2}{*}{ Reference } & \multicolumn{2}{|c|}{$\alpha_{0^{+}}$} & \multicolumn{2}{|c|}{$\alpha_{2^{+}}$} & \multicolumn{2}{|c|}{$\alpha_{4^{+}}$} & \multicolumn{2}{|c|}{$\alpha_{6^{+}}$} \\
\hline & $\mathrm{E}_{\alpha}, \mathrm{keV}$ & $\mathrm{P}_{\alpha}, \%$ & $\mathrm{E}_{\alpha}, \mathrm{keV}$ & $\mathrm{P}_{\alpha}, \%$ & $\mathrm{E}_{\alpha}, \mathrm{keV}$ & $\mathrm{P}_{\alpha}, \%$ & $\mathrm{E}_{\alpha}, \mathrm{keV}$ & $\mathrm{P}_{\alpha}, \%$ \\
\hline \multicolumn{9}{|l|}{${ }^{246} \mathrm{Cm}:$} \\
\hline [20] & 5387 & 78 & 5345 & 22 & & & & \\
\hline [21] & 5387 (4) & $78(5)$ & $5345(5)$ & $22(5)$ & & & & \\
\hline [22] & 5385 & 79 & 5342 & 21 & & & & \\
\hline [23] & $5386.5(10)$ & $82.2(12)$ & $5343.5(10)$ & $17.8(12)$ & & & & \\
\hline Present work & $5386(3)$ & $79.08(22)$ & $5342(3)$ & $20.9(4)$ & $5242(3)$ & $0.020(2)$ & & \\
\hline \multicolumn{9}{|l|}{${ }^{250} \mathrm{Cf}:$} \\
\hline [24] & $6024(5)$ & 83 & 5980 & 17 & & & & \\
\hline$[25]$ & $6029(2)$ & 84.7 & $5987(5)$ & 15 & $5890(5)$ & 0.3 & $5736(7)$ & $\sim 0.01$ \\
\hline Present work & $6030(3)$ & $82.6(1)$ & $5988(3)$ & $17.11(13)$ & $5891(3)$ & $0.283(15)$ & $5742(3)$ & 0.007 (2) \\
\hline
\end{tabular}

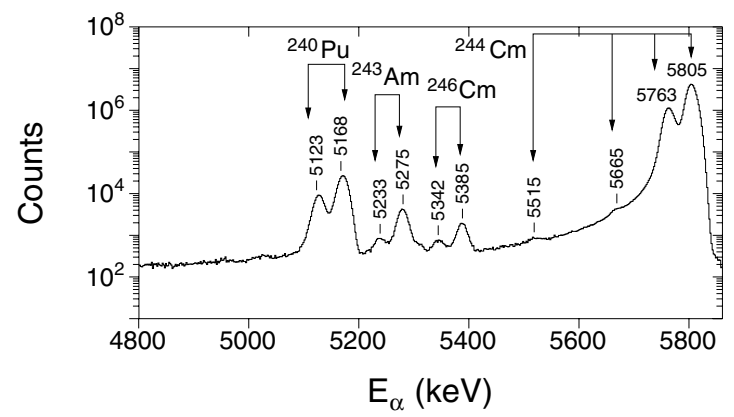

Fig. 2. Alpha-particle spectrum of a ${ }^{244} \mathrm{Cm}$ source measured with a $4.5 \mathrm{~cm}^{2}$ PIPS detector at a solid angle of $6.0 \%$ - accumulation time was $24.5 \mathrm{~d}$.

the 106.12-keV $\gamma$-ray $\left({ }^{239} \mathrm{Pu}\right)$ was found to be unchanged in a number of measurements performed over a long period of time. There is essentially no uncertainty involved in the determination of the growth time $\left(t_{c}\right)$, since the sources were dated at the time of the mass separation. Overall, we estimate the total systematic uncertainty to be below $0.1 \%$.

\subsection{Alpha-particle and gamma-ray emission probabilities}

The $\alpha$-decay emission probabilities of ${ }^{250} \mathrm{Cf}$ and ${ }^{246} \mathrm{Cm}$ were determined by integrating the areas under the alpha peaks of spectra collected at small solid angle $(\Omega=0.06 \%)$ in order to eliminate alpha-electron coincidence summing. The resulting values are presented in table 3 , together with results from earlier studies. Measured $\mathrm{P}_{\alpha}$ values for the main alpha groups of ${ }^{246} \mathrm{Cm}$ in the present work differ by $3.8 \%\left(\alpha_{0^{+}}\right)$and $17.4 \%$ $\left(\alpha_{2^{+}}\right)$from those of Shatinskii [23] which were determined by means of a magnetic spectrograph. Given the high isotopic purity of the source used in the present measurements and the fact that we have carefully checked for possible alpha-electron coincidence summing and pile-up effects that might impact on the measured intensities, a high-degree of confidence can be placed in our results. Using ${ }^{246} \mathrm{Cm}$ and ${ }^{250} \mathrm{Cf}$ source spectra, we have determined essentially identical values for the intensity ratio of the two main ${ }^{246} \mathrm{Cm}$ alpha groups of $\mathrm{P}_{\alpha}\left(\alpha_{2^{+}}\right) / \mathrm{P}_{\alpha}\left(\alpha_{0^{+}}\right)=0.264$ (5) and 0.265 (8), respectively. Our ${ }^{250} \mathrm{Cf} \alpha$-decay emission probabilities for the $\alpha_{0^{+}}$and $\alpha_{2^{+}}$ groups differ by about $2.5 \%$ and $14.1 \%$, respectively, from those measured by Baranov et al. [25], albeit no uncertainties were reported in their work. We have also determined a much more precise value of $\mathrm{P}_{\alpha}=0.007$ (2)\% for the transition to the $6^{+}$level of the ground state band of ${ }^{246} \mathrm{Cm}$, compared with an approximate value of $0.01 \%$ reported previously by Baranov et al. [25]. Differences between the alpha intensities measured with semiconductor detectors and magnetic spectrographs have been noted in past studies of other actinide sources, and were attributed to systematic uncertainties inherent in the latter technique [26]. We are investigating these descrepancies in detail for other nuclides that have been previously studied by means of magnetic spectrographs and for which we have access to mass-separated sources.

Measurements of the $\gamma$-ray emission probabilities of ${ }^{237} \mathrm{~Np}$ and daughter ${ }^{233} \mathrm{~Pa}$ have been initiated in an underground laboratory using a mass separated ${ }^{237} \mathrm{~Np}$ source. The main aim is to resolve differences between previous experimental results of the emission probability of the $28.6-\mathrm{keV} \gamma$ ray in the decay of ${ }^{233} \mathrm{~Pa}$ and to improve further the accuracy of the $\gamma$-ray emission probabilities for other transitions following the decay of ${ }^{233} \mathrm{~Pa}$ and ${ }^{237} \mathrm{~Np}$. Additional measurements are planned 
with a purified ${ }^{233} \mathrm{~Pa}$ source that will be produced by milking ${ }^{237} \mathrm{~Np}$ parent activity and carrying out a radiochemical separation prior to spectroscopic analysis to avoid interference from the higher intensity $29.4-\mathrm{keV}$ (E1) transition of ${ }^{237} \mathrm{~Np}$.

\section{Concluding remarks}

Well-defined decay data are essential pre-requisites in the detection and accurate quantification of fissile radionuclides. Parameters of greatest relevance include actinide half-lives, branching fractions, and $\alpha$-particle and $\gamma$-ray energies and emission probabilities - their highly credible definition to good accuracy provides the means of monitoring their presence, behavior and transport in nuclear facilities as well as any clandestine movement and usage. The primary aim of the Coordinated Research Project on "Updated Decay Data Library for Actinides" is the preparation of a dedicated database for a range of nuclear applications. Measurements have been undertaken and will continue to determine specific decay characteristics of the more inadequately defined radionuclides. However, most IAEA-CRP efforts are now expected to focus on the comprehensive evaluation of the decay scheme data for the agreed set of actinides and their decay products.

This work was supported by the US Department of Energy, Office of Nuclear Physics, under Contract No. DE-AC02-06CH11357 and was undertaken with the assistance of the Nuclear Data Section of the International Atomic Energy Agency under the auspices of the Coordinated Research Project "Updated Decay Data Library for Actinides". The authors are thankful to the Office of Basic Energy Sciences, US Department of Energy, through the transplutonium element production facilities at the Oak Ridge National Laboratory, for the use of ${ }^{246} \mathrm{Cm}$ and ${ }^{250} \mathrm{Cf}$ material. The authors wish to thank all members of the IAEA-CRP for their advice during the course of the work.

\section{References}

1. International Atomic Energy Agency, Proc. Advisory Group Meeting on Transactinium Isotope Nuclear Data, 3-7 Nov. 1975, Karlsruhe, Germany, IAEA-186, IAEA, Vienna, Austria (1976).

2. International Atomic Energy Agency, Decay Data of the Transactinium Nuclides, Technical Reports Series No. 261, IAEA, Vienna, Austria (1986).

3. S. Hofmann, G. Munzenberg, Rev. Mod. Phys. 72, 733 (2000).

4. A.L. Nichols, Appl. Radiat. Isot. 55, 23 (2001).

5. M.A. Kellett, F.G. Kondev, A.L. Nichols, Int. Conf. Radionuclide Metrology and Its Applications (ICRM2007), 3-7 Sept. 2007, Cape Town, South Africa.
6. M.A. Kellett, Summary Report of the First Research Coordination Meeting on Updated Decay Data Library for Actinides, 17 - 19 October 2005, INDC(NDS)-0479, IAEA, Vienna, Austria (2006).

7. M.A. Kellett, Summary Report of the Second Research Coordination Meeting on Updated Decay Data Library for Actinides, 28 - 30 March 2007, INDC(NDS)-0508, IAEA, Vienna, Austria (2007).

8. F.G. Kondev, I. Ahmad, J.P. Greene, M.A. Kellett, A.L. Nichols, Appl. Radiat. Isot. 65, 335 (2007).

9. I. Ahmad, F.G. Kondev, J.P. Greene, M.A. Kellett, A.L. Nichols, Nucl. Instrum. Meth. Phys. Res. A (in press).

10. J. Lerner, Nucl. Instrum. Meth. 102, 373 (1972).

11. W.W. Strohm, Int. J. Appl. Radiat. Isot. 35, 155 (1984).

12. M.M. Bé, V. Chisté, C. Dulieu, E. Browne, V. Chechev, N. Kuzmenko, R. Helmer, A.L. Nichols, E. Schonfeld, R. Dersch, Table of Radionuclides $-A=151$ - 242, Vol. 2, Monographie BIPM-5, 2004.

13. M.M. Bé, V. Chisté, C. Dulieu, E. Browne, C. Baglin, V. Chechev, N. Kuzmenko, R. Helmer, F.G. Kondev, D. MacMahon, K.B. Lee, Table of Radionuclides $-A=3$ - 244, Vol. 3, Monographie BIPM-5, 2006.

14. J.R. Stokely, C.E. Bemis Jr., R.D. Baybarz, R. Eby, J. Inorg. Nucl. Chem. 35, 3369 (1973).

15. D.N. Metta, H. Diamond, F.R. Kelly, J. Inorg. Nucl. Chem. 31, 1245 (1969).

16. K.W. MacMurdo, R.M. Harbour, R.W. Benjamin, J. Inorg. Nucl. Chem. 33, 1241 (1971).

17. V.G. Polyukhov, G.A. Timofeev, V.V. Kalygin, P.A. Privalova, Radiokhimiya 24, 490 (1982); Sov. J. Radiochem. 24, 408 (1982).

18. V.G. Polyukhov, G.A. Timofeev, P.A. Privalova, V.Ya. Garbeskiriya, A.P. Chetverikov, Radiokhimiya 19, 507 (1977); Sov. J. Radiochem. 19, 414 (1977).

19. J.E. McCracken, J.R. Stokely, R.D. Baybarz, C.E. Bemis, Jr., R. Eby, J. Inorg. Nucl. Chem. 33, 3251 (1971).

20. L.M. Belov, B.S. Dzhelepov, R.B. Ivanov, A.S. Krivokhatskii, V.G. Nedovesov, V.P. Chechev, Radiokhimiya 5, 394 (1963); Sov. J. Radiochem. 5, 362 (1963).

21. B.S. Dzhelepov, R.B. Ivanov, V.G. Nedovesov, V.P. Chechev, JETP 45, 1360 (1963); Sov. Phys. JETP 18, 937 (1963).

22. S.A. Baranov, Yu.P. Radionov, V.M. Kulakov, V.M. Shatinskii, Yad. Fiz. 4, 1108 (1966); Sov. J. Nucl. Phys. 4, 798 (1967).

23. V.M. Shatinskii, At. Energy 56, 245 (1984); Sov. J. At. Energy 56, 282 (1984).

24. F. Asaro, F.S. Stephens Jr., B.G. Harvey, I. Perlman, Phys. Rev. 100, 137 (1955).

25. S.A. Baranov, V.M. Shatinskii, V.M. Kulakov, Yad. Fiz. 11, 701 (1970); Sov. J. Nucl. Phys. 11, 393 (1970).

26. I. Ahmad, Nucl. Instrum. Meth. Phys. Res. A 223, 319 (1984). 